\title{
ngl9: A Third MADS Box Gene Expressed in Alfalfa Root Nodules
}

\author{
Joseph C. Zucchero, Michal Caspi, and Kathleen Dunn \\ Department of Biology, Boston College, Chestnut Hill, MA 02467 U.S.A. \\ Submitted 19 January 2001; Accepted 25 August 2001.
}

\begin{abstract}
Expression of MADS box genes has previously been localized to the infected cells of alfalfa (Medicago sativa) root nodules. These genes represent the first putative transcription factors to be identified in nodules and are hypothesized to be involved in a signal transduction pathway initiated by the intracellular bacterium. The eventual activation of specific target genes defines pertinent characteristics of this nitrogen-fixing differentiated cell. In this study, we identify a third nodule MADS box gene, $n g l 9$, and demonstrate that the DNA-binding activity of its protein product is dependent on the presence of a second MADS box protein, NMH7. Despite previous results to the contrary, both genes are expressed in the early stages of flower development, further strengthening the premise that nodule developmental programming may capitalize upon existing developmental cascades.
\end{abstract}

As part of a continuing investigation into root nodule development, we have looked for regulatory mechanisms that might be conserved between other developmental processes in plants, such as those regulating flower development. Many of the regulatory genes involved in flower development encode proteins with a conserved 58-amino acid, DNA-binding domain (Sommer et al. 1990; Yanofsky et al. 1990). This DNA-binding domain from these putative transcription factors is conserved among numerous organisms and, in plants, has been termed the MADS box (M-mcml; A-ag; D-def A; S-srf) (Schwarz-Sommer et al. 1990). A common feature to many members of this class of regulatory proteins is that they often function in the determination of cell fate in response to environmental signals (Riechmann and Meyerowitz 1997).

Previously, we cloned and characterized two MADS box genes that are expressed in nodules, $n m h 7$ and $n m h c 5$ (Heard and Dunn 1995; Heard et al. 1997). In situ hybridization using the conserved MADS box domain from $n m h 7$ showed that expression of the nodule MADS box genes is localized to the infected cells. Thus, our working hypothesis has been that these

Corresponding author: Kathleen Dunn; Telephone: +1-617-552-3217; E-mail: dunnm@bc.edu

Current address of Michal Caspi: The Weizmann Institute of Science, Department of Molecular Genetics, P.O. Box 26, Rehovot 76100 Israel.

Nucleotide and/or amino acid sequence data for $n g l 9$ are available in the GenBank database under the accession number AF335473. putative transcriptional regulators are involved in a signal transduction pathway initiated by the infecting bacterium. We anticipate that the presence of MADS box proteins results in the activation of specific target genes whose products directly or indirectly define the differentiated state of the infected cell.

Here, we report the cloning and characterization of a third MADS box gene, $n g l 9$, and demonstrate that the DNA-binding activity of its corresponding protein requires the presence of NMH7. The tissue specificity of these nodule genes further strengthens our contention that the regulation of flower development may serve as a useful paradigm for nodule development.

MADS box proteins are known to form homodimers or heterodimers as a requisite for DNA binding. Based on its proposed amino acid sequence, NMH7 is favored to form heterodimers but not homodimers; NMHC5 appears capable of forming either. Previous binding experiments have suggested a homodimeric interaction using truncated forms of NMHC5 and the CArG element from yeast, the conserved binding sequence for MADS box proteins (Heard et al. 1997). Since NMH7 did not appear to form a specific complex with a CArG oligonucleotide in the mobility shift assay as a homodimer, or as a heterodimer with NMHC5, we have predicted that a third MADS box protein is likely involved in dimer formation. A good candidate for such a partner would be the alfalfa homologue of the Antirrhinum majus gene encoding GLO, which has been shown to form dimers with the NMH7 homologue, DEFA (Trobner et al. 1992).

A polymerase chain reaction (PCR) approach was employed to identify a glo homologue expressed in alfalfa root nodules. Based on the Antirrhinum glo DNA sequence, two degenerate oligonucleotides within the conserved MADS box domain were designed and used in a PCR amplification with alfalfa nodule RNA. The resulting alfalfa cDNA product showed high homology to the MADS box domain of glo. Using a nested primer within the alfalfa MADS box domain and an oligo(dT)-adapter primer, a second round of PCR amplification with nodule RNA generated a PCR product that began at the 34th nucleotide and ended in the $3^{\prime}$ untranslated region (UTR). Using the extreme $5^{\prime}$ coding sequence from glo and the alfalfa nucleotide sequences from the first PCR product, remaining nucleotides were added. Since the first eight residues are encoded by the glo gene, they remain unconfirmed for the alfalfa homologue. MADS box domains are generally conserved, but the N-terminal region of the MADS box is extremely conserved within subfamilies and, thus, is likely to be the correct sequence. A similar approach was used for the 
cDNA cloning of $n m h 7$ and $n m h c 5$ (Heard and Dunn 1995; Heard et al. 1997). While subsequent sequencing of the corresponding $n m h 7$ genomic clone revealed two additional amino acids at the $\mathrm{N}$ terminus, there were no amino acid changes from the region of the original priming sequence (Kirby et al. 1998).

Conceptual translation of the open reading frame yielded a polypeptide sequence with 212 amino acid residues (Fig. 1). Like other MADS box genes, ngl9 encodes the highly conserved MADS box, structurally conserved $\mathrm{K}$ and I domains, and a C-terminal region (Purugganan et al. 1995; Rounsley et al. 1995). Based on its sequence information, the predicted translation product of $n g l 9$ belongs to the AP3 (DEFA)/PI
(GLO) subfamily. The putative NGL9 shows the highest degree of overall homology to the Petunia MADS protein pMADS2 $(72 \%)$ and shares a $70 \%$ overall identity with GLO. NGL9 shows amino acid conservation in each of the three functional domains, MADS, I, and K, when compared with other members of its subfamily (Fig. 2). In addition, NGL9 also has a stretch of conserved amino acids at the K/C-terminus junction. These conserved sequences are also found in PI, GLO, and a number of other MADS box proteins (Riechmann and Meyerowitz 1997). As with all MADS box proteins, the C terminus shows the greatest variation, and NGL9 has no obvious structural characteristics that would indicate that this region has a functional significance. However, using chimeric

\section{ATGGGAAGAGGAAAGATTGAGATCAAGAGGATTGAGAACTCAAGCAACAGACAAGTTACA 60 $\begin{array}{llllllllllllllllllll}\mathbf{M} & \mathbf{G} & \mathbf{R} & \mathbf{G} & \mathbf{K} & \mathbf{I} & \mathbf{E} & \mathbf{I} & \mathbf{K} & \mathbf{R} & \mathbf{I} & \mathbf{E} & \mathbf{N} & \mathbf{S} & \mathbf{S} & \mathbf{N} & \mathbf{R} & \boldsymbol{Q} & \mathbf{V} & \mathbf{T}\end{array}$ \\ 61 TATTCAAAGAGAAAAAGTGGATTTAAAGAAAGTAACGAAATCAATGTTCTATGTGAT 120 $\begin{array}{llllllllllllllllllll}\mathbf{Y} & \mathbf{S} & \mathbf{K} & \mathbf{R} & \mathbf{K} & \mathbf{S} & \mathbf{G} & \mathbf{I} & \mathrm{L} & \mathbf{K} & \mathbf{K} & \mathbf{A} & \mathbf{N} & \mathbf{E} & \mathbf{I} & \mathbf{N} & \mathbf{V} & \mathbf{L} & \mathbf{C} & \mathbf{D}\end{array}$}

101 GCTCAAGTTTCCACTATCATCTTTGCTCCTTCTGGAAAAATGCATGAATACATTAGCCCT 180 $\begin{array}{llllllllllllllllllll}\mathbf{A} & \mathbf{Q} & \mathbf{V} & \mathbf{S} & \mathbf{T} & \mathbf{I} & \mathbf{I} & \mathbf{F} & \mathbf{A} & \mathbf{P} & \mathbf{S} & \mathbf{G} & \mathbf{K} & \mathbf{M} & \mathbf{H} & \mathbf{E} & \mathbf{Y} & \mathbf{I} & \mathbf{S} & \mathbf{P}\end{array}$

181 TCTACCACGTTGATTGATATGCTGGAGAGATATCAAAAAGCATCTGGAAAGAGGCTTTgG 240 $\begin{array}{llllllllllllllllllll}S & T & T & L & I & D & M & L & E & R & Y & Q & K & A & S & G & K & R & L & W\end{array}$

241 GATGCAAAACATGAGAACTTAAGGAATGAAATTGAGAAACTCAAGAAAGAGAATGAGGAC 300 $\begin{array}{llllllllllllllllllll}\mathrm{D} & \mathrm{A} & \mathrm{K} & \mathrm{H} & \mathrm{E} & \mathrm{N} & \mathrm{L} & \mathrm{R} & \mathrm{N} & \mathrm{E} & \mathrm{I} & \mathrm{E} & \mathrm{K} & \mathrm{L} & \mathrm{K} & \mathrm{K} & \mathrm{E} & \mathrm{N} & \mathrm{E} & \mathrm{D}\end{array}$

301 ATGGAAATTAAGCTCAGGCACTTGAAGGGAAAGGATATCAACACACTAAACTACAAGAAG 360 $\begin{array}{lllllllllllllllllllll}M & E & I & K & L & R & H & L & K & G & K & D & I & N & T & L & N & Y & K & K\end{array}$

361 TTGATGAGCTTAGAGGATGTCTTAGAAAATGGTCTCCTCACTATCCGTGACAAACAGATG 420 $\begin{array}{llllllllllllllllllll}L & M & S & L & E & D & V & L & E & N & G & L & L & T & I & R & D & K & Q & M\end{array}$

421 GAAGTATACCAGATGGTAACGAGAAACGACAAGATCTTGGAGGAGGAGAATAGGGAACTT 480 $\begin{array}{lllllllllllllllllllll}E & V & Y & Q & M & V & T & R & N & D & K & I & L & E & E & E & N & R & E & L\end{array}$

481 AATTTTATCCTCCAGCAACAGCATATGGCAGTGGAAGGTGGAGAGAAATGGATTATAATG 540 $\begin{array}{llllllllllllllllllll}N & F & I & L & Q & Q & Q & H & M & A & V & E & G & G & E & K & W & I & I & M\end{array}$

541 ATGAATTTGATGAGAGTAGTGGAAGTGATTTCAATTCCCAGATGCCATTTTCCTTTCGTC 600 $\begin{array}{llllllllllllllllllll}M & N & L & M & R & V & V & E & V & I & S & I & P & R & C & H & F & P & F & V\end{array}$

601 TTCAGCCTATGCAGCTTCACTCACTACTTCAGCTTTAAATTCCACCCAAAATTAAGACTT 660 $\begin{array}{lllllllllllllllllllll}F & S & L & C & S & F & T & H & Y & F & S & F & K & F & H & P & K & L & R & L\end{array}$

661 TTAGTAACTATATATGTTGATTTATGATAATTGTAATAAAGAAGCTGAATCTGGGTTTTT 720

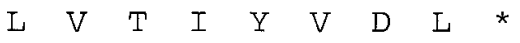

721 GAGGTTACATTACATTATATATGCGTGTTTAATTTAACATAGTATATGACCCTTGGACTT 780 GCACTTTCACTTAAAAAAAA

Fig. 1. Nucleotide and deduced amino acid sequence of $n g l 9$. The MADS box domain is shown in bold. $n g l 9$ cDNA was sequenced in both directions using the Sequenase II kit according to the manufacturer's instructions (United States Biochemical, Cleveland, OH, U.S.A.). The open reading frame is 636 nucleotides long, encoding a putative protein of 212 amino acids. The underlined sequence at the $5^{\prime}$ end is the Antirrhinum majus glo primer sequence and thus unconfirmed for the alfalfa $n g l 9 \mathrm{cDNA}$. The underlined sequence at 696 is the polyadenylation site. 
genes in an in vivo functional assay, a role for the $\mathrm{C}$ terminus in flower development was demonstrated for its Arabidopsis homologue, PI (Krizek and Meyerowitz 1996). Recent findings of a ternary complex between GLO and other MADS box proteins also involved regions within the $\mathrm{C}$ terminus, prompting the authors to suggest a mechanism by which these regulatory proteins may facilitate expression of downstream genes (Egea-Cortines et al. 1999).

The ability of NGL9 and its predicted dimer partner NMH7 to bind a specific sequence of DNA was examined using the CArG motif found in the upstream region of STE2, a target gene for the yeast MADS box protein MCM1 (Passmore et al. 1989). Several MADS box proteins found in plants have been shown to bind the STE2 CArG element in vitro (SchwarzSommer et al. 1992). $n g l 9$ and $n m h 7$ were cloned into the pET expression vector, and the nodule MADS box proteins were purified from separate preparations of inclusion bodies (Fig. 3). NGL9 and NMH7 were subsequently mixed together, in- cubated with a ${ }^{32} \mathrm{P}$-labeled STE2 probe, and assayed for complex formation on native acrylamide gels. As shown in Figure 4, only samples containing both NMH7 and NGL9 were able to retard the movement of the STE2 CArG element. Extracts containing NMH7 or NGL9 alone or the pET vector without insert were unable to shift the DNA probe. The specificity of the NMH7-NGL9 interaction with the STE2 CArG element was demonstrated by showing that a mutated STE2 probe with a disrupted CArG box failed to bind the complex (data not shown).

These results are consistent with the DNA binding of an NMH7-NGL9 heterodimer. This was predicted by the structure of the I domains and because the corresponding homologues, AP3/PI from Arabidopsis and PI/DEFA from Antirrhinum also bind DNA as heterodimers. The I domain, thought to be important in dimer stability, demonstrates a clustered pattern of charged residues at the $C$ terminus of the region. In NGL9, these residues carry the opposite charge

$\mathbf{N H}_{4}^{+}$\begin{tabular}{|l|l|l|l|}
\hline MADS-box & I-domain & K-box & \\
\hline
\end{tabular}

\section{A. MADS box}

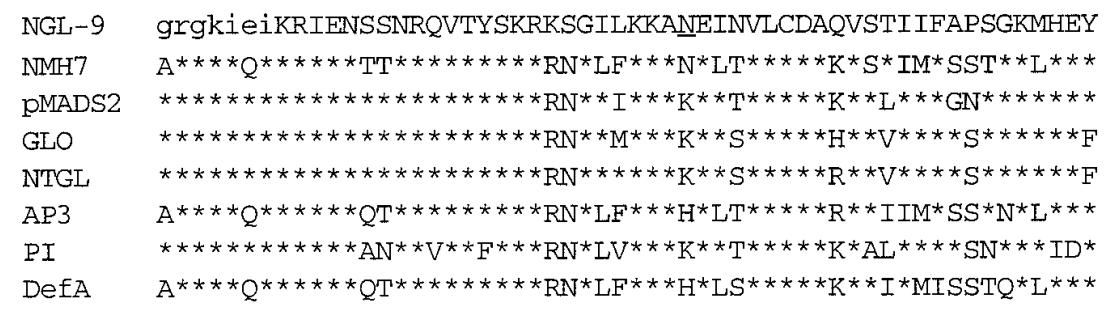

\section{B. I domain}

\begin{tabular}{|c|c|}
\hline \multirow[t]{2}{*}{ NGL-9 } & ISPSTTLIDMLERYQKASGKRLWDAKHENLR \\
\hline & + \\
\hline NMH7 7 & ---STK--QFFDQYQTTVGIDLWNSHYENMQ \\
\hline PMADS2 & 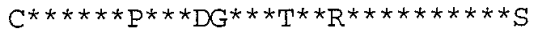 \\
\hline GLO & 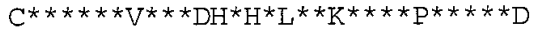 \\
\hline NTGLO & $--S^{\star} * T^{*} V^{*} I^{*} \mathrm{DQ} Q^{*} \mathrm{H}^{*} \mathrm{LT} \mathrm{T}^{*} \mathrm{R}^{* * * * * *}$ \\
\hline P3 & ISPNTTTKEIVDLYQTISDVDVWATQYERMQ \\
\hline & CCPSMDLGAMLDQYQKLSGKKLWDAKHENLS \\
\hline & $\star \star \star * T * A T K Q L F D Q Y Q K A V G V D L W S S H Y E K M Q$ \\
\hline
\end{tabular}

\section{C. $\mathrm{K}$ box}

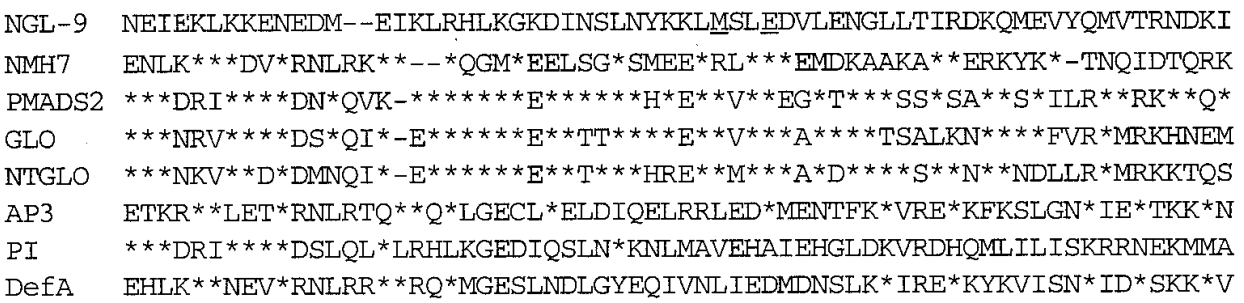

Fig. 2. An alignment of $n g l 9$ to MADS box regions of related MADS box genes: A, MADS box domain; B, I domain; and $\mathbf{C}$, K box domain. Schematic drawing of NGL9 shows relative position of the various domains. Plus (+) and minus (-) charge distribution of residues within the I domain is shown for NGL9 and NMH7. 
from that found in $\mathrm{NMH7}$, indicating the ability of these two MADS box proteins to form heterodimers. The $\mathrm{K}$ box, believed to be important in recruiting accessory proteins, forms the predicted amphipathic helical structure, with other hydro-
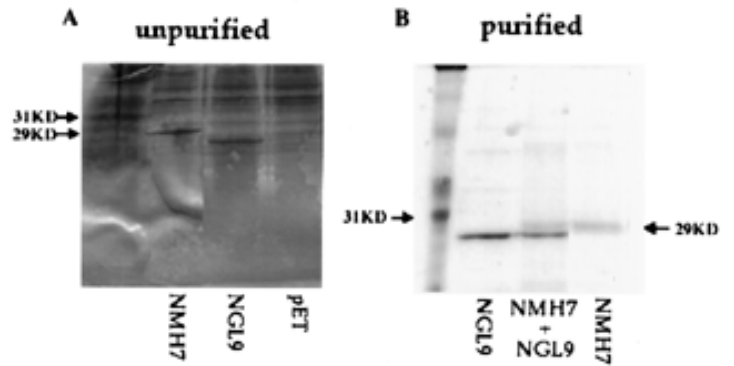

Fig. 3. Coomassie-stained gel of proteins extracted from the pET bacterial expression system (Novagen, Madison, WI, U.S.A.). Induced cells containing pET-nmh7, pET-ngl9, and pET(control) were harvested. The proteins were subjected to the purification process as described by Huang and colleagues (1995). Extracts of NMH7 and NGL9 were mixed before the addition of urea. The protein extracts were run on a $10 \%$ sodium dodecyl sulfateacrylamide gel, stained with Coomassie blue for $1 \mathrm{~h}$, and destained overnight. A, Protein extracts before purification. B, Partially purified proteins.

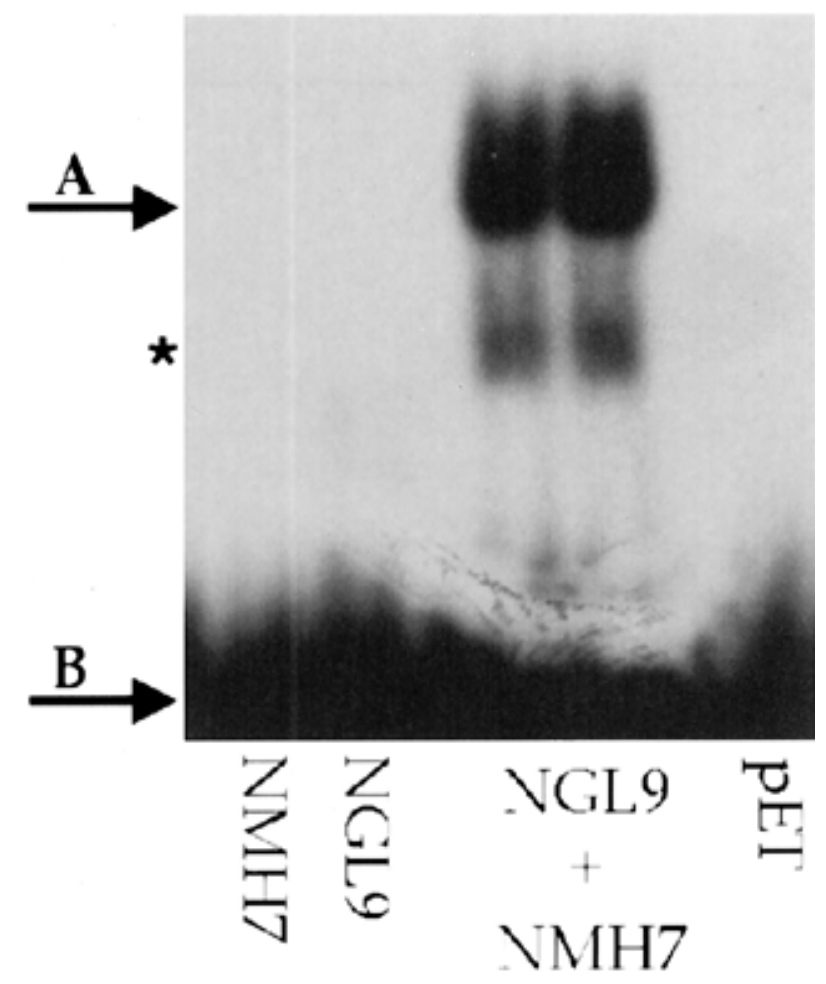

Fig. 4. Electrophoretic mobility shift assay (EMSA) of NMH7 and NGL9 with a CArG-like element from the yeast STE2 promoter. The partially purified protein extracts $(10 \mu \mathrm{g})$ were added to approximately $10^{4} \mathrm{cpm}$ by the ${ }^{32}$ P-labeled STE2 probe and assayed for their DNA-binding activity on native acrylamide gels. A, The protein DNA complex. B, Free probe * A nonspecific complex with the CArG probe routinely forms in all EMSA reactions including those in which the specific expressed protein is not present. The highest level of intensity is seen in those reactions in which complex formation has occurred (Heard and Dunn 1995; Heard et al. 1997). Here, the nonspecific complex appears to be below the level of detection in all lanes except where both proteins are present, perhaps indicative of a more highly purified protein or oligonucleotide preparation. phobic amino acids replacing the leucines. In Arabidopsis, heterodimer interaction between the AP3 and PI proteins was demonstrated in solution using modified proteins and immunoprecipitation (Goto and Meyerowitz 1994). The binding of DNA by the AP3-PI heterodimer was also shown to require the $\mathrm{N}$ terminus of the $\mathrm{K}$ region, in addition to the MADS and I domains (Riechmann et al. 1996). ngl9 belongs to the same family of genes, and the boundaries of the protein domains as shown in Figure 2 are consistent with the domain configuration from Arabidopsis. However, similar studies with NGL9 and NMH7 have not been done, neither have any experiments that would verify a direct interaction between the two MADS box proteins.

The expression of $n g l 9$ in flower and nodule tissues of alfalfa was examined through the use of reverse-transcriptionPCR. For purposes of comparison, $n m h 7$ was also included. Total RNA was isolated from nodule and flower, and cDNA was made using a $3^{\prime}$ oligo(dT)-adapter primer (3' RACE system; formerly Gibco/BRL, now Invitrogen, Carlsbad, CA, U.S.A.). Transcripts that contained a MADS box domain were amplified using a $5^{\prime}$ primer designed to the MADS box region of $n g l 9$ or $n m h 7$ in conjunction with the $3^{\prime}$ oligo(dT)-adapter primer. PCR products were then subjected to gel electrophoresis, transferred to a nylon membrane, and probed with a 100-bp oligonucleotide designed to the $3^{\prime}$ UTR of $n g l 9$ or $n m h 7$.

As shown in Figure 5A, the probe to the $3^{\prime}$ UTR of $n g l 9$ detected a band of approximately $0.8 \mathrm{~kb}$ in both nodule and flower lanes, while the $3^{\prime}$ UTR probe for $n m h 7$ detected a $0.9-\mathrm{kb}$ band, also in the nodule and flower lanes (Fig. 5B). The presence of $n g l 9$ RNA in nodules is consistent with the expression pattern for $n m h 7$ (Fig. 5B). Interestingly, previous work in this lab did not detect $n m h 7$ expression in flower tissue (Heard and Dunn 1995). It is possible that $n m h 7$ is transiently expressed during flower development and was not detected in the earlier experiments, in which the flower tissue used was considered older. This would be consistent with the expression patterns of ap3 and pi in Arabidopsis, in which neither gene is expressed in later stages of flower development (Goto and Meyerowitz
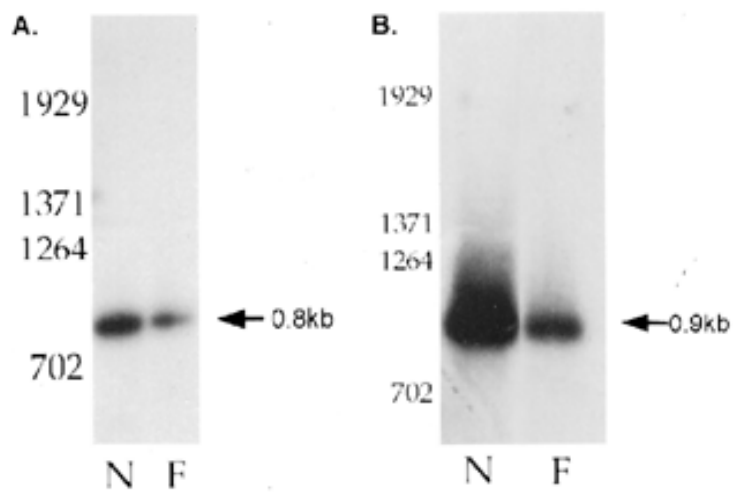

Fig. 5. Reverse-transcription-polymerase chain reaction (RT-PCR) analysis of MADS box transcripts from total RNA isolated from nodule $(\mathrm{N})$ and flower (F). Size markers from lambda cut with BstEII (3' RACE system; formerly Gibco/BRL, now Invitrogen, Carlsbad, CA, U.S.A.). RT-PCR products $(2 \mu \mathrm{g})$ were separated on a $1 \%$ agarose gel, transferred to a nylon membrane (Hybond-N), and probed with a ${ }^{32} \mathrm{P}$-labeled probe designed to the $3^{\prime}$ untranslated region of $\mathbf{A}$, $n g l 9$ or $\mathbf{B}, n m h 7$ as described previously (Heard and Dunn 1995). 
1994). Expression of the nodule MADS box gene $n m h c 5$ was retested and, as reported previously (Heard et al. 1997), found in nodule tissue but not in flower. Preliminary data also suggest that $n g l 9$ expression is not found in either root or leaf tissue, a pattern consistent with the expression of other alfalfa MADS box genes. These results need to be verified.

In summary, we have cloned $n g l 9$, the third MADS box gene expressed in alfalfa root nodules. Based on mobility shift assays and its homology to GLO and PI, we believe that NGL9 will emerge as the dimer partner for the nodule MADS box protein NMH7. However, the expression profile for $n g l 9$ is not complete. While our earlier in situ hybridizations indicated that MADS box genes were localized to the infected cells, it's not known if the $n m h 7$ MADS box probe was able to detect $n g l 9$ transcripts (Heard and Dunn 1995). Thus, it is not possible to verify coexpression at the cellular level. The interaction of MADS box proteins amongst themselves or with potential accessory proteins is still not well characterized. Nonetheless, the presence of $n g l 9$ and $n m h 7$ transcripts in both alfalfa nodule and flower tissues and the general role that MADS box genes are thought to play in the determination of cell fate encourages us to speculate about overlapping developmental programs. New information from ongoing plant genome projects combined with more exhaustive screening procedures could facilitate a comparison between the development of flowers and nodules and the roles that $n g l 9$ and $n m h 7$ play in each of these processes.

\section{ACKNOWLEDGMENTS}

This work was supported by a grant from the National Science Foundation (IBN-9305007).

\section{LITERATURE CITED}

Egea-Cortines, M., Saedler, H., and Sommer, H. 1999. Ternary complex formation between the MADS-box proteins SQUAMOSA, DEFICIENS and GLOBOSA is involved in the control of floral architecture in Antirrhinum majus. EMBO (Eur. Mol. Biol. Organ.) J. 18:5370-5379.

Goto, K., and Meyerowitz, E. M. 1994. Function and regulation of the Arabidopsis floral homeotic gene PISTILLATA. Genes Dev. 8(13): 1548-1560.

Heard, J., and Dunn, K. 1995. Symbiotic induction of a MADS-box gene during development of alfalfa root nodules. Proc. Natl. Acad. Sci. U.S.A. 92:5273-5277.
Heard, J., Caspi, M., and Dunn, K. 1997. Evolutionary diversity of symbiotically induced nodule MADS box genes: Characterization of $n m h C 5$, a member of a novel subfamily. Mol. Plant-Microbe Interact. 10:665-676.

Huang, H., Tudor, M., Weiss, C. A., Hu, Y., and Ma, H. 1995. The Arabidopsis MADS-box gene AGL3 is widely expressed and encodes a sequence-specific DNA-binding protein. Plant Mol. Biol. 28:549-567.

Kirby, C., Heard, J., Carroll, S., Ganter, G., Lesher, J., and Dunn, K. 1998. The genomic sequence of $n m h 7$, a MADS box gene whose expression has been localized to the infected cells of alfalfa (Medicago sativa) root nodules. Plant Physiol. 116:1604.

Krizek, B. A., and Meyerowitz, E. M. 1996. Mapping the protein regions responsible for the functional specificities of the Arabidopsis MADS domain organ-identity proteins. Proc. Natl. Acad. Sci. U.S.A. 93: 4063-4070.

Passmore, S., Elble, R., and Tye, B.-K. 1989. A protein involved in minichromosome maintenance in yeast binds a transcriptional enhancer conserved in eukaryotes. Genes Dev. 3:921-935.

Purugganan, M. D., Rounsley, S. D., Schmidt, R. J., and Yanofsky, M. F. 1995. Molecular evolution of flower development: Diversification of the plant MADS-box regulatory gene family. Genetics 140:345-356.

Riechmann, J. L., and Meyerowitz, E. M. 1997. MADS domain proteins in plant development. Biol. Chem. 378:1079-1101.

Riechmann, J. L., Krizek, B. A., and Meyerowitz, E. M. 1996. Dimerization specificity of Arabidopsis MADS domain homeotic proteins APETALA1, APETALA3, PISTILLATA and AGAMOUS. Proc. Natl. Acad. Sci. U.S.A. 93:4793-4798.

Rounsley, S. D., Ditta, G. S., and Yanofsky, M. F. 1995. Diverse roles for MADS box genes in Arabidopsis development. Plant Cell 7:12591269.

Schwarz-Sommer, Z., Huijser, P., Nacken, W., Saedler, H., and Sommer, H. 1990. Genetic control of flower development: Homeotic genes in Antirrhinum majus. Science 250:931-936.

Schwarz-Sommer, Z., Hue, I., Huijser, P. I., Flor, P. J., Hansen, R., Tetens, F., Lonnig, W.-E., Saedler, H., and Sommer, H. 1992. Characterization of the homeotic MADS-box gene deficiens: Evidence for DNA binding and autoregulation of its persistent expression throughout flower development. EMBO (Eur. Mol. Biol. Organ.) J. 11:251-263.

Sommer, J., Beltram, J.-P., Huijser, P., Pape, H., Lonnig, W.-E., Saedler, H., and Schwarz-Sommer, Z. 1990. deficiens, A homeotoic gene involved in the control of flower morphogenesis in Antirrhinum majus: The protein shows homology to transcription factors. EMBO (Eur. Mol. Biol. Organ.) J. 9:605-613.

Trobner, W., Ramirez, L., Motte, P., Hue, J., Huijser, P., Lonning, W. E., Saedler, H., Sommer, H., and Schwarz-Sommer, Z. 1992. GLOBOSA: A homeotic gene which interacts with DEFICIENS in the control of Antirrhinum floral organogenesis. EMBO (Eur. Mol. Biol. Organ.) J. 11:4693-4704.

Yanofsky, M. F., Ma, H., Bowman, J. L., Drew, G. N., Felfman, K. A., and Meyerowitz, E. M. 1990. The protein encoded by the Arabidopsis homeotic gene agamous resembles transcription factors. Nature 346: 35-39. 